\title{
PAPERS
}

\section{A fractional order parallel control structure tuned with meta-heuristic optimization algorithms for enhanced robustness}

\author{
Vishal Goyal ${ }^{*}$, Puneet Mishra ${ }^{* *}$, Aasheesh Shukla*, \\ Vinay Kumar Deolia*, Aarti Varshney*
}

\begin{abstract}
This paper studies an improved fractional order parallel control structure (FOPCS) for enhancing the robustness in an industrial control loop having a first order process with dead time along with its tuning aspects. Since inclusion of fractional order calculus also increase the number of parameters to be determined for a particular control loops, tuning becomes an essential task. Four different tuning methods are considered to optimize the gains of parallel control structure (PCS) and FOPCS. Integral of time weighted absolute error for servo and regulatory problems along with overshoot value have been considered for performance evaluation. Extensive simulation studies including change in setpoint and mismatch in processmodel parameters have been carried out. On the basis of these studies, it was observed that FOPCS tuned by backtracking search algorithm, outperformed all other controllers in terms of considered performance measures.

K e yw ords: fractional order calculus, meta-heuristic optimization algorithms, parallel control structure, PID controller, robustness, tuning
\end{abstract}

\section{Introduction}

Efficient control of industrial systems has been of paramount importance in all the process industries in order to achieve maximum profitability and productivity from the plant. The concern about the efficient control can range from human safety to various involved economic benefits depending upon the nature of industry. However, irrespective of the ultimate objective of industrial plant, different process variables are needed to be controlled at low level so as to finally achieve the primary objectives. These low level control operations usually include servo, regulation and robustness problems. A conventional control structure (CCS), employing a single controller is frequently used in the industries to accomplish these objectives. However, these objectives are seldom achieved with maximum efficiency using CCS. To achieve most out of a control strategy in terms of servo, regulatory and robustness performance, a parallel control structure (PCS) has been reported in literature [1]. This PCS essentially decouples the setpoint tracking and disturbance rejection capabilities of the control scheme by separately designing dedicated controllers. These separate controllers can be tuned judiciously to achieve better system performance in associated operating regime.

Conventionally PCS uses classical proportional-integral-derivative (PID) controllers for its implementation. The reason for the same is their simple structure, easy to understand behavior, ability to deal with few nonlinearities like actuator saturation, and integrator windup. The PID controller is the most dominating controller in industries and more than $90 \%$ of all control loops use them $[2,3]$. It has also been reported that there are only 5-10\% of industrial loops which employ controllers other than PID [4]. However, despite having numerous advantages, there are some constraints in which PID controllers cannot provide an efficient solution for the control of process variable. The ubiquitously used classical linear PID controllers fail to provide adequate efficiency if they are operated upon nonlinear, uncertain or time-varying systems $[5,6]$. Also, the nonlinearity and uncertainty in an industrial system is unavoidable. If a system is nonlinear in nature, its parameters' value may change with operating points of the process. In such situations PID controllers fail to portray themselves as a feasible and best control algorithm. Moreover, these have a fixed control structure and hence their robustness is compromised. Further, it can be easily verified that a single PID controller can only provide either, better setpoint tracking or better load regulation. To overcome these issues of parametric uncertainty, different algorithms have been reported in literature. However, industries are still in phase of evaluating these recently developed controllers, in view of their tuning issues, complex structure and objective involved. Another potential solution which can deal with these issues is inclusion of fractional order calculus in control algorithms. The fractional order PID (FOPID) controllers are generalized PID controllers, where the strengths of the integration and derivative may be different from unity. The FOPID controller, without compromising with the simplicity of the PID control, are claimed to provide improved robustness along with all other advantages of the PID controller. Many instances have been reported in literature where fractional order calculus has provided bet-

* Department of Electronics and Communication Engineering, GLA University, Mathura, India, ** Department of Electrical and Electronics Engineering, Birla Institute of Technology and Science - Pilani, Pilani campus, Jhunjhunu, Rajasthan, India, aasheesh.shukla@gla.ac.in 
ter robustness and control performance, for uncertain and nonlinear systems [7-9]. A fractional order fuzzy controller is proposed for the control of a distillation column and improved robustness and control performance is achieved [6].Another instance can be seen of use fractional order control for the control an antilock braking system [10]. The increased use of fractional order control is spreading widely and different applications are being reported in literature [12-15]. It may be noted that, FOPID controller has more flexibility than a classical PID controller by the virtue of two additional parameters in contrast to a classical PID controller.

It may be observed on the basis of conducted literature survey, that inclusion of fractional order calculus can provide greater robustness and deal with the parametric uncertainties in a better manner. However, the industrial control requirements include three main characteristics, ie setpoint tracking capability, disturbance rejection capability and robustness. First two of these requirements can be achieved using a parallel control structure which is specifically designed for the decoupling of servo and regulatory actions. However, third requirement ie robustness can be enhanced with the inclusion of the fractional order operators in PCS. Overall, it may be thought that a PCS along with fractional order calculus can provide greater robustness along with decoupled servo and load regulation actions. This paper is an effort to study the same and investigates the enhancement in the performance for the control structure in terms of better control operation. A fractional order PCS (FOPCS) [23] is studied in this paper regarding the tuning of its parameters. The FOPCS structure uses FOPID controllers to provide better robustness along with decoupled tracking and regulatory actions. The performance of the studied control structure is evaluated quantitatively using three measures viz. integral of time weighted absolute error (ITAE) for setpoint tracking as well as disturbance rejection, overshoot (OS) and integral of absolute rate of controller output(IARCO). A first order plus dead time (FOPDT) system is used as a test plant, since behavior of most of the industrial processes can be approximated using FOPDT model. Further, to design an efficient FOPCS, the tuning of the gains of FOPID controller is achieved using two global optimization algorithms viz. differential evolution (DE) algorithm and backtracking search algorithm (BSA). Two other established tuning algorithms, direct synthesis (DS) and gain margin - phase margin (GM-PM) method, have also been employed to compare the efficiencies of the studied FOPCS and conventional PCS.

\section{Description of fractional order parallel control structure}

PCS is a dedicated control strategy consisting of two simultaneously running control loops [1]. The two control loops effectively decouple the setpoint tracking and load regulation. However, the third requirement of robustness is seldom achieved in conventional PCS structure.
Robustness requirement in a control loop is essentially desired due to parametric uncertainty; on account of either embedded process nonlinearities, wrong estimation of plant dynamics, or ageing effects etc. A robust controller will help in keeping the similar performance of the control operation as in the nominal case of no plant-model mismatch. The robustness in a conventional PCS can be enhanced by proper tuning of the controller parameters, but at the expense of reduced tracking and regulation performance. There are different strategies through which a robust control scheme can be designed however it is always desired that the industrial nature of the controller do not get changed. Recently, fractional order controllers, which are an extension of the integer order controllers, are now finding their place in industry. It is claimed that they can provide a greater robustness in comparison of their integer order counterpart in terms of better performances, for fractional order systems [16], higher order systems [17], for non-minimum phase system, systems with long time delays [18-20], and systems with nonlinearities $[21,22]$.

The most common form of a fractional order PID controller is shown in (3), which involves an integrator of order $\lambda$ and a differentiator of order $\mu$; where, $\lambda, \mu \in \mathfrak{R}$. The transfer function for a fractional order controller is

$$
G_{c}(s)=K_{p}\left(1+\frac{1}{T_{i} s^{\lambda}}+T_{d} s^{\mu}\right)
$$

where, $K_{p}$ is the proportional constant gain, $T_{i}$ is the integral time and $T_{d}$ is the derivative time. To design integer order controller for conventional PCS in this work, $\lambda$ and $\mu$ were set to unity.

Figure 1 shows the structure of analyzed FOPCS [23], where the controllers for setpoint tracking $G_{c}^{S T}\left(s^{\mu}\right)$ and disturbance rejection $G_{c}^{D R}\left(s^{\mu}\right)$ have a PID structure but are now extended to fractional order. In other words, the conventional PCS which decouples the setpoint tracking and disturbance rejection actions can now be thought as to provide robustness to handle the parametric uncertainty.

It may be noted from Fig. 1 that, $G_{p}$ is the process which is to be controlled, $y$ is process variable. Disturbance $d$ is acting directly on the process and is added to the process variable. $u$ is the control input applied to the process and is generated through the combination of the two FOPID controllers $G_{c}^{S T}\left(s^{\mu}\right)$ and $G_{c}^{D R}\left(s^{\mu}\right)$. However, this decoupled action can only be achieved if the process dynamics are estimated accurately by an appropriate means. In this work, an estimated transfer function $G_{m}(s)$ is considered for the implementation of PCS and FOPCS. Further, it may be inferred from Fig. 1, that

$$
\begin{aligned}
y=\frac{G_{p}\left(1+G_{c}^{D R} G_{m}\right)}{G_{m}\left(1+G_{c}^{D R} G_{p}\right)}\left[\frac{G_{c}^{S T} G_{m}}{1+G_{c}^{S T} G_{m}}\right] r & \\
+ & {\left[\frac{1}{1+G_{c}^{D R} G_{p}}\right] d }
\end{aligned}
$$




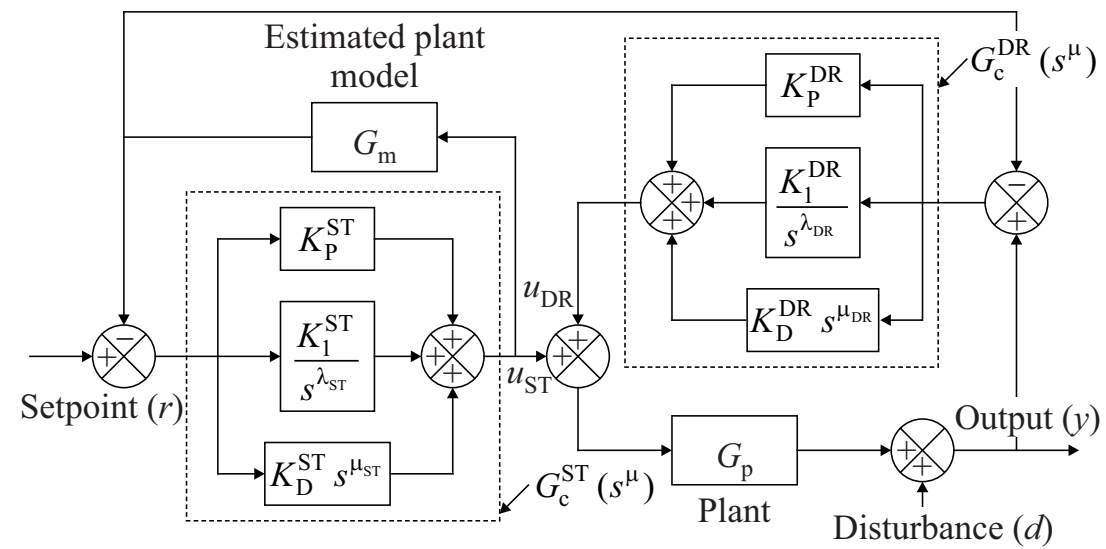

Fig. 1. Fractional order parallel control structure block diagram [23]

If $G_{p}=G_{m}$, then (2) reduces to

$$
y=\left[\frac{G_{c}^{S T} G_{m}}{1+G_{c}^{S T} G_{m}}\right] r+\left[\frac{1}{1+G_{c}^{D R} G_{m}}\right] d
$$

It can be easily interpreted from $(2)$, that $G_{C}^{S T}\left(s^{\mu}\right)$ is responsible for setpoint tracking of the process variable and $G_{C}^{D R}\left(s^{\mu}\right)$ can handle the disturbances and parametric uncertainties. The gains and fractional order dynamics of these controllers can be independently tuned to have decoupled tracking and regulatory actions, however it will occur in nominal conditions only where plant and its estimated mathematical model matches to a great extent. In case of a mismatch, a robustness requirement will be originated and hence fractional order calculus can help in achieving the desired robustness [23]. Many methods have been presented in literature to mimic the behavior of a fractional order operator. These methods include power series expansion (PSE), curve fitting method, optimization based designs on the basis of frequency response of fractional order operators, Oustaloup's recursive approximation (ORA) and so on. However, an acceptable and widely used method see ORA is the one which has been chosen for this work due to its simplicity yet powerful design which closely approximates the desired fractional order operators in a given frequency range. In ORA method, the poles and zeros of a higher order filter are recursively estimated and the structure if the fractional order operator can be given as

$$
s^{\mu}=H(s), \mu \in \Re .
$$

This function can be approximated as follows [6]

$$
s^{\mu} \approx K^{\prime} \prod_{k=-N}^{k=N} \frac{s+\omega_{z_{k}}}{s+\omega_{p_{k}}}
$$

where, $K^{\prime}$ is gain, $\left.\omega_{(} z_{k}\right)$ and $\left.\omega_{(} p_{k}\right)$ are the zeros and poles and can be calculated as

$$
\omega_{p_{k}}=\omega_{L}\left(\frac{\omega_{H}}{\omega_{L}}\right)^{\frac{k+N+\frac{1}{2}(1+\mu)}{2 N+1}}
$$

and

$$
\omega_{z_{k}}=\omega_{L}\left(\frac{\omega_{H}}{\omega_{L}}\right) \frac{k+N+\frac{1}{2}(1-\mu)}{2 N+1} .
$$

The range of angular frequencies in which the fractional order operators are estimated in this work is $0.01 \mathrm{rad} / \mathrm{s}$ to $100 \mathrm{rad} / \mathrm{sec}$. This range is the most common range for the systems widely used in process control application. Also, the choice of $N$ plays a vital role in the performance of fractional order approximation. Larger value of $\mathrm{N}$ permits good approximation, fewer ripples in the magnitude and phase behavior but computational complexity is increased. The current research work considers the value of $N$ as three.

Further, the studied FOPCS using fractional order controllers and conventional PCS have been tested for their performance on an FOPDT system. Since, most of the industrial processes can be modeled using FOPDT dynamics, all the performance comparative studies have been performed on this system. Also, it may be noted that both, the conventional PCS and FOPCS, uses different controllers and thereby require different parameter values for their implementation. The values of their parameters significantly affect the performance of the control loop and hence tuning methods to find optimum values play a critical role. The subsequent section discusses the same aspect for PCS and FOPCS in detail.

\section{Controller parameter optimization}

There are different types of tuning methods which can be categorized into two parts viz. traditional and intelligent methods. Conventional tuning methods such as Ziegler Nichols, Direct Synthesis method, Gain Margin Phase Margin method, Aström-Hägglund method, quarter decay ratio method, are still being used in industrial environments. However these methods are not suitable for the systems which possess nonlinear characteristics, uncertain behaviour or are used in non-conventional control structures such as PCS itself. Moreover, these traditional tuning methods also fail to minimize a customized performance index. For such type of scenarios, recently a field of engineering, meta-heuristic optimization techniques has been evolved. These techniques find a global 
optimal solution for a complex multimodal optimization problem either using bio-inspired mechanisms or physical laws etc. Also, these algorithms are free from gradient search, and hence are more efficient for providing a global optimal solution. Different global optimization algorithms such as genetic algorithm (GA), differential evolution (DE), particle swarm optimization algorithm, cuckoo search algorithm, backtracking search algorithm (BSA), harmony search algorithm, grey wolf optimizer, and many more algorithms are available in literature [2430]. These algorithms are widely used for solving problems related to different areas and control engineering is not an exception. More many instances controller parameters cannot be set heuristically for a nonlinear system or in cases where the number of parameters is more. Other than that, standard tuning rules as in literature cannot be applied uniformly to every controller or control structure. This fact has led this work to adopt global optimization algorithms viz. DE and BSA for the controller tuning. The results are then compared with two traditional tuning algorithms ie DS and GM-PM.

\subsection{Performance evaluation index}

In order to tune the controller parameters using considered optimization algorithm, it is necessary to design an appropriate objective function. This objective function must comprise of index related to desired control performance. In this work, the optimization problem takes a form of aggregation of three performance parameters, ie ITA $E_{\mathrm{ST}}$, ITA $E_{\mathrm{DR}}$, and $\mathrm{OS}$, where, ITAE $E_{\mathrm{ST}}$ is integral time weighted absolute error for setpoint tracking response, $I T A E_{\mathrm{DR}}$ is integral time weighted absolute error for disturbance rejection and OS is taken as maximum OS in the tracking response. The objective function which is to be minimized, is the algebraic summation of all the three parameters and can be written as

$$
\begin{aligned}
& (J)=I T A E_{\mathrm{ST}}+I T A E_{\mathrm{DR}}+\mathrm{OS} \\
& \text { with: } I T A E=\int_{t_{o}}^{t_{f}} t|e(t) \mathrm{d} t|
\end{aligned}
$$

here, $t_{o}$ and $t_{f}$ are the initial and final time of the study for a particular case $i e$ either setpoint tracking or disturbance rejection study. As already discussed, the plant on which the performance of the PCS and FOPCS is to be tested is an FOPDT system, the transfer function of the system under nominal conditions, is considered as

$$
G_{p}(s)=G_{m}(s)=\frac{2}{3 s+1} e^{-s} .
$$

For tuning of the controller parameters, a setpoint of unity is applied to the system at $t=0 \mathrm{~s}$, and a step disturbance of magnitude 0.5 is applied at $t=10 \mathrm{~s}$. It is worth mentioning here that controller output was deliberately kept in a range of $[-5,5]$ using a saturator since controller output has to be limited to mimic real world scenario. Both DE and BSA were used for the parameter optimization of controllers $G_{c}^{S T}(s)$ and $G_{c}^{D R}(s)$ with maximum iterations of 100 and population size of 20 . For the optimization of PCS using integer order controllers, the range of $K_{p}^{*}, K_{i}^{*}$ and $K_{d}^{*}\left({ }^{*}-\mathrm{ST} / \mathrm{DR}\right)$ is kept same as in the case of FOPCS structure for FOPID controller.

\subsection{Tuning of PCS and FOPCS}

Figure 2 shows objective function vsiteration curve for $\mathrm{DE}$ and BSA. It can be easily seen from this figure that both DE and BSA have achieved their best in specified number of iterations. It can also be observed from Figure2 that the integer order controllers, for both DE and $\mathrm{BSA}$, provide higher objective function values than their respective fractional order counterpart.

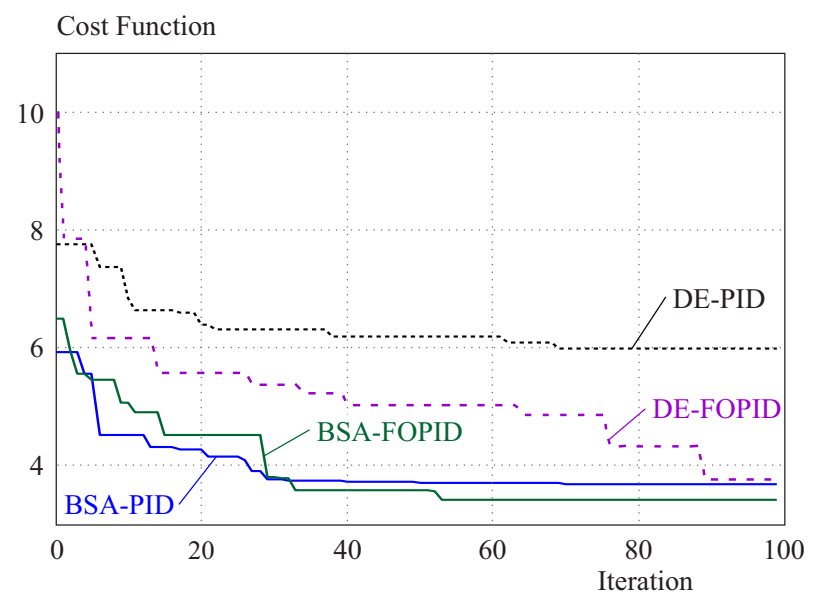

Fig. 2. Objective function vs iteration curve

The minimum value of J for DE-PID, BSA-PID, DEFOPID and BSA-FOPID are 5.982, 3.680, 3.753 and

Table 1. Tuned gains for PCS and FOPCS

\begin{tabular}{lcccccc}
\hline Method & $K_{p}^{S T}$ & $K_{i}^{S T}$ & $K_{d}^{S T}$ & $K_{p}^{D R}$ & $K_{i}^{D R}$ & $K_{d}^{D R}$ \\
\hline GMPM-PID & 1.177 & 0.392 & 0.00 & 0.785 & 0.261 & 0.00 \\
DS-PID & 0.750 & 0.250 & 0.375 & 0.750 & 0.250 & 0.375 \\
DE-PID & 0.634 & 0.199 & 0.103 & 0.680 & 0.200 & 0.120 \\
DE-FOPID & 0.794 & 0.290 & 0.144 & 0.771 & 0.288 & 0.158 \\
BSA-PID & 0.849 & 0.241 & 0.125 & 0.789 & 0.233 & 0.021 \\
BSA-FOPID & 0.725 & 0.264 & 0.187 & 0.873 & 0.277 & 0.108 \\
\hline
\end{tabular}


3.409 respectively. The achieved gains for each case of optimization are listed in Tab. 1.

Further, the gains of the controllers are also achieved using traditional tuning algorithms viz. DS and GMPM [1]. The values of different parameters achieved using these techniques are also listed in Tab. 1.

\section{Simulation results}

This section deals with investigating the performance of conventional PCS and studied FOPCS tuned by GM-PM, DS, DE, and BSA. These simulation studies include testing of the efficacies of the control structures for nominal case, nominal case with different setpoint, parameter mismatch case $i e$ mismatch in steady state gain, dead time and time constant estimation. Each study include setpoint tracking as well as disturbance rejection studies which are run for $20 \mathrm{~s}$, in which initial $10 \mathrm{~s}$ are for servo problem and at $t=10 \mathrm{~s}$, a step disturbance is applied to the system with a magnitude of 0.5. The considered simulation studies have been carried out using Runge Kutta - 4 ODE solver with a step size of $0.01 \mathrm{~s}$ on a personal computer having Intel Core $^{\mathrm{TM}}$ i5 processor, with 4 GB RAM on LabVIEW ${ }^{\mathrm{TM}}$ platform.

\subsection{Nominal case $G_{m}=G_{p}$}

In this study a nominal model is considered ie no plant model mismatch condition. Figure 3 shows the response of different control structure tuned by considered algorithms for setpoint tracking and disturbance rejection responses. It may be observed from Fig. 3 that all the controllers were able to achieve the specified setpoint and tracked it in steady state. However, transient responses for all considered controller are different. It can be inferred from Fig. 3, that the PCS having PID controller tuned using GM-PM method is exhibiting maximum OS.

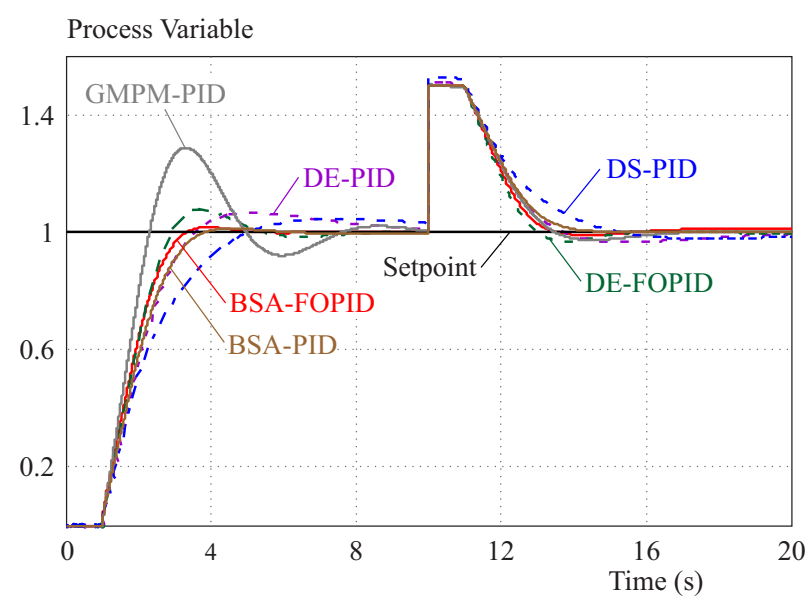

Fig. 3. Process variable variation for nominal case

Further, the PID controller tuned using DS, DE, and BSA are also having overshoot but BSA tuned PID is showing a better performance among all the considered PID controllers. However, FOPCS, tuned using BSA, is showing best performance among all with smallest OS along with fastest settling time and outperforms every other considered controller for servo as well as regulatory problem.

Table 2. Performance comparison of PCS and FOPCS in nominal case

\begin{tabular}{lccccc}
\hline Method & ITAE $_{S T}$ & ITAE $_{\mathrm{DR}}$ & OS $(\%)$ & IARCO & $J$ \\
\hline GMPM-PID & 4.113 & 1.403 & 28.67 & 2.767 & 5.803 \\
DS-PID & 4.462 & 2.330 & 4.545 & 685.3 & 6.838 \\
DE-PID & 3.994 & 1.987 & 0.025 & 200.0 & 5.982 \\
DE-FOPID & 2.486 & 1.192 & 7.497 & 2.555 & 3.753 \\
BSA-PID & 2.396 & 1.271 & 1.211 & 158.5 & 3.680 \\
BSA-FOPID & 1.973 & 1.419 & 1.580 & 4.215 & 3.409 \\
\hline
\end{tabular}

Quantitatively also, objective function value $J$ is minimum for BSA-FOPID at a value of 3.409 with $I T A E_{\mathrm{ST}}$, $I T A E_{\mathrm{DR}}$ and $\mathrm{OS}$ as $1.973,1.419$ and 0.015 respectively. For more elaborated quantitative comparison between the performances of the controllers, Table 2 lists all the performance index values. Also, another performance parameter ie integral of absolute rate of controller output (IARCO) is calculated and depicted in Tab. 2. The importance of IARCO is in the fact that it indicates the smoothness in the controller output. If the controller output is smooth then the life span of the different control element will be increased, since there will be lesser excursion on the control loop elements. It may be clearly seen from the quantitative comparison of considered controllers that the BSA-FOPID controller employed in PCS is performing best with minimum objective function. In practical scenarios, the tuned control structure may experience a different environment from the one where the controller was originally tuned. Thereby it is essential to test the efficacy of the controller in different circumstances in which the controller may be used. In order to assess, the performance of FOPCS, some plant model mismatch cases and a change in setpoint have been considered. Subsequent section addresses same simulation studies.

\subsection{Nominal case with different setpoint value $(r=1.5)$}

It is quite possible that a controller tuned for one setpoint may be used for other setpoint. In this work, same issue has been addressed and all the considered controllers are again applied to the nominal system, but with a different setpoint value. Since, it may be possible that a control system, which is tuned for a better setpoint tracking, has proportional kick and with an increased setpoint value, the effect of proportional kick would be more severe. In view of this, it is important that the controller be tested for different setpoint value from the one which was used for the tuning purpose. Figure 4 shows the process variable variations after applying step input with magnitude of 1.5 at $t=0$. In this study, no disturbance was injected to the control loop. It may be observed from Fig. 4 that the response of the BSA tuned FOPID controller is still 
Table 3. Performance comparison of controllers for setpoint $(r)=1.50$

\begin{tabular}{lcccc}
\hline Method & ITAE $_{\text {ST }}$ & OS (\%) & IARCO & $J$ \\
\hline GMPM-PID & 6.170 & 28.671 & 3.366 & 6.45 \\
DS-PID & 6.738 & 4.622 & 660.445 & 6.78 \\
DE-PID & 5.979 & 0.053 & 182.989 & 5.97 \\
DE-FOPID & 3.729 & 7.497 & 2.178 & 3.80 \\
BSA-PID & 3.668 & 0.873 & 236.97 & 3.67 \\
BSA-FOPID & 3.107 & 0.624 & 5.32 & 3.11 \\
\hline
\end{tabular}

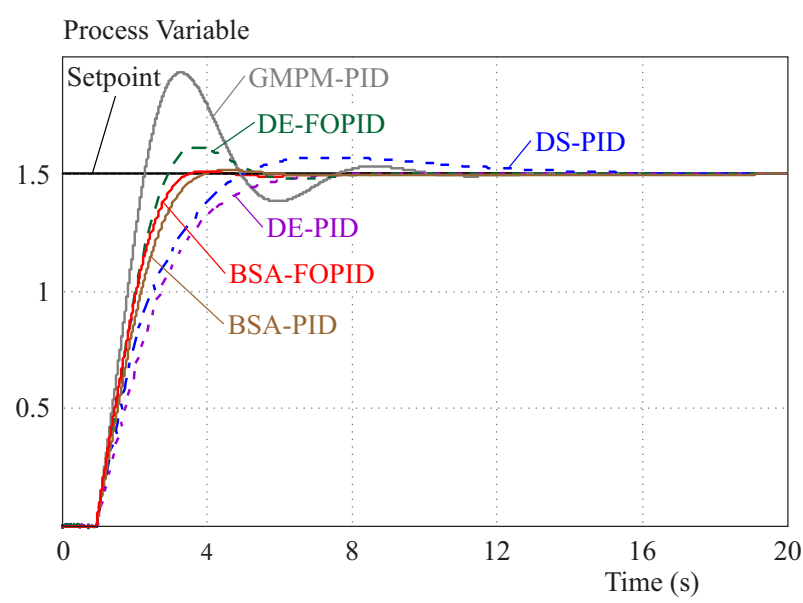

Fig. 4. Process variable variation for $r=1.50$

best among all the controllers with minimum ITAE $E_{\mathrm{ST}}$ of 3.107. It may be noted here that the performance trend is same as achieved in tuning scenario and the relevant performance measures are listed in Tab. 3. The performance assessment parameters considered in this study are only OS and ITAE $E_{\mathrm{ST}}$.

\subsection{Plant - model mismatch case}

As already discussed, industrial processes are often represented by a FOPDT model. Most of the time, it may happen that the estimated model do not represent the plant behavior exactly either due to improper modeling or due to plant - model mismatch. Causes for plant - model mismatch is usually due to wrong estimation of the plant dynamics, or drift in plant parameters due to ageing effect or wear and tear; or may be due to nonlinearity. In nonlinear systems, the plant parameters change significantly as the operating point changes and hence the plant behavior may be quite different from the estimated FOPDT model. In view of this, a control structure must be tested for robustness. This subsection deals with the same investigation and various robustness testing studies have been carried out.

\subsubsection{Mismatch in process static gain}

In this investigation, it is assumed that the actual plant gain and its estimated gain are not exactly same. A mismatch of $5 \%$ is considered. This will affect the decoupling performance as well as the individual servo and disturbance rejection performance. To cope up with such scenarios, the controller must be robust enough to prevent degradation in performance for setpoint tracking and disturbance rejection. Moreover to achieve this, the controller action must not be very aggressive. In this study, the mismatched condition is assumed which can be given as

$$
G_{m}(s)=\frac{2}{3 s+1} e^{-s} \neq G_{p}(s)=\frac{2.1}{3 s+1} e^{-s} .
$$

Table 4. Performance comparison of controllers for $5 \%$ change in static gain

\begin{tabular}{lccccc}
\hline Method & ITAE $_{\text {ST }}$ & ITAE $_{\text {DR }}$ & OS (\%) & IARCO & $J$ \\
\hline GMPM-PID & 4.541 & 1.400 & 33.336 & 2.902 & 6.275 \\
DS-PID & 4.353 & 2.259 & 4.928 & 685.839 & 6.661 \\
DE-PID & 3.594 & 1.773 & 0.193 & 200.108 & 5.369 \\
DE-FOPID & 2.573 & 1.225 & 10.128 & 2.659 & 3.900 \\
BSA-PID & 2.337 & 1.208 & 2.919 & 158.577 & 3.575 \\
BSA-FOPID & 2.008 & 1.478 & 3.897 & 4.298 & 3.525 \\
\hline
\end{tabular}

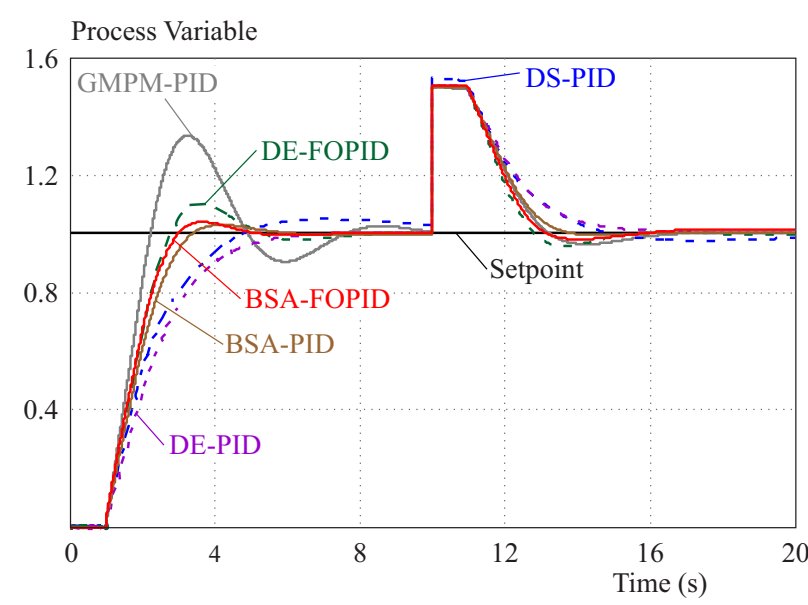

Fig. 5. Process variable variation for $5 \%$ change in static gain

To evaluate the performance of FOPCS, setpoint tracking and disturbance rejection studies have been performed using same inputs as in the nominal case. Figure4 shows the tracking performance of the considered controllers. Again, the PID controller tuned with GMPM method is showing largest OS. However, the FOPCS structure tuned with BSA is providing the optimum performance with very less overshoot, optimum rise time, along with better disturbance rejection in comparison with all other considered controllers. For quantitative comparison the objective function $(J)$ is considered along with the value of IARCO and is listed in Tab. 4. It may be noted from the quantitative comparison provided in Tab. 4, that the value of $J$ is minimum for BSA tuned FOPCS at a value of 3.525. A closer value of 3.575 is also achieved by BSA tuned PCS, but at the expense of increase controller action and this variation in controller action is very aggressive which should be avoided. The 


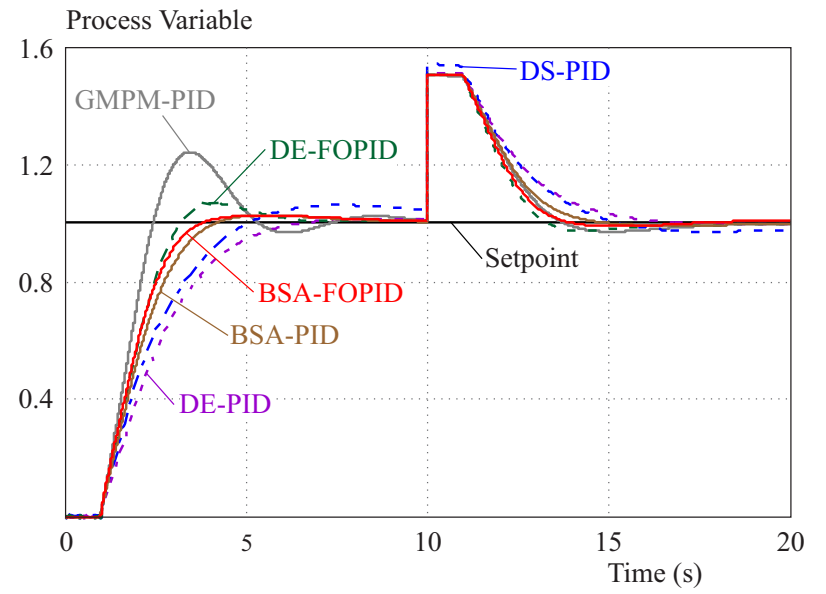

Fig. 6. Process variable variation for $10 \%$ change in time constant

Table 5. :Performance comparison of controllers for $10 \%$ change in time constant

\begin{tabular}{lccccc}
\hline Method & ITAE $_{\text {ST }}$ & ITAE $_{\mathrm{D}}$ & OS $(\%)$ & IARCO & $\mathrm{J}$ \\
\hline GMPM-PID & 3.667 & 1.807 & 24.033 & 2.592 & 5.715 \\
DS-PID & 5.179 & 2.746 & 6.038 & 684.511 & 7.986 \\
DE-PID & 4.478 & 2.146 & 1.368 & 199.925 & 6.638 \\
DE-FOPID & 2.754 & 1.528 & 6.569 & 2.442 & 4.348 \\
BSA-PID & 3.019 & 1.669 & 2.498 & 158.495 & 4.714 \\
BSA-FOPID & 2.751 & 1.437 & 2.405 & 4.165 & 4.213 \\
\hline
\end{tabular}

aggression in the controller action is quantified with the help of IARCO value, which is 158.577 for BSA tuned PCS in comparison of mere 4.298 for BSA tuned FOPCS. It may also be noted that the control performance of other controllers such as GM-PM tuned PCS, DS tuned PCS, DE tuned PCS, and DE tuned FOPCS are worse as the objective function values of these controllers along with IARCO values are high in comparison to BSA tuned FOPCS.

4.3.2 Mismatch in process time constant

For second case of plant - model mismatch, time constant of the process has been considered. The value of time constant of process may significantly affect the phase margin of the system and thereby plant stability or control performance. This fact necessitates the need of testing of the designed controllers for such type of conditions. In this work, also this issue has been investigated and the robustness of the controller is tested. In this study, the process and estimated model are taken as follows

$$
G_{m}(s)=\frac{2}{3 s+1} e^{-s} \neq G_{p}(s)=\frac{2}{3.3 s+1} e^{-s}
$$

It may be observed here, that there is a change in time constant value of $10 \%$ and is increased from $3.00 \mathrm{~s}$ to $3.30 \mathrm{~s}$. Figure 6 shows the variation in process variable for this case study. It may be observed from this figure that the GM-PM tuned PID controller is again showing largest OS with a objective function value of 5.715. On the contrary, both FOPCS structures, ie tuned by DE and BSA, are showing lesser OS and objective function values as well. It may be noted that, FOPCS have very less objective function value which clearly shows the robustness increment in the control loop. However, BSA tuned FOPCS is depicting best performance among all and has an objective function value of 4.213 in comparison of its close competitor ie DE tuned FOPCS which has a $J$ value of 4.348 . Table 5 shows the quantitative comparison among all the control structures.

It is worth mentioning here that BSA tuned PCS is also showing a comparable performance with that of the BSA tuned FOPCS. However, this similar level of performance is achieved by an aggressive control action as depicted by the value of IARCO. The value of IARCO for BSA tuned PCS is 158.495 against mere 4.165 which is achieved by BSA tuned FOPCS for even lesser objective function value. There are also spikes in the control action of BSA tuned PCS controller which may result in damaging final control element and can even reduce the longevity of final control elements.

\subsection{3 $\mathrm{Mismatch}$ in process dead time}

Process dead time is a significant cause for the instability in the control loop. Wrong estimation of the process dead time may lead to erroneous tuning of controller gains and may provide reduced level of control performance. It is essential that a controller be robust enough to handle variation in the process dead time. It has already been demonstrated that the inclusion of fractional order controller in the parallel control structure will provide a better level of robustness in the system. This investigation deals with another case of the robustness testing in which the process dead time is different from the estimated one.

Table 6. Performance comparison of controllers for $5 \%$ change in dead time

\begin{tabular}{lccccc}
\hline Method & ITAE $_{\text {ST }}$ & ITAE $_{\text {DR }}$ & OS (\%) & IARCO & J \\
GMPM-PID & 4.510 & 1.548 & 31.235 & 2.829 & 6.371 \\
DS-PID & 4.568 & 2.379 & 4.881 & 685.381 & 6.997 \\
DE-PID & 3.914 & 1.938 & 0.103 & 201.036 & 5.853 \\
DE-FOPID & 2.808 & 1.337 & 9.990 & 2.658 & 4.246 \\
BSA-PID & 2.590 & 1.323 & 2.869 & 158.58 & 3.942 \\
BSA-FOPID & 2.213 & 1.562 & 3.802 & 4.251 & 3.813 \\
\hline
\end{tabular}




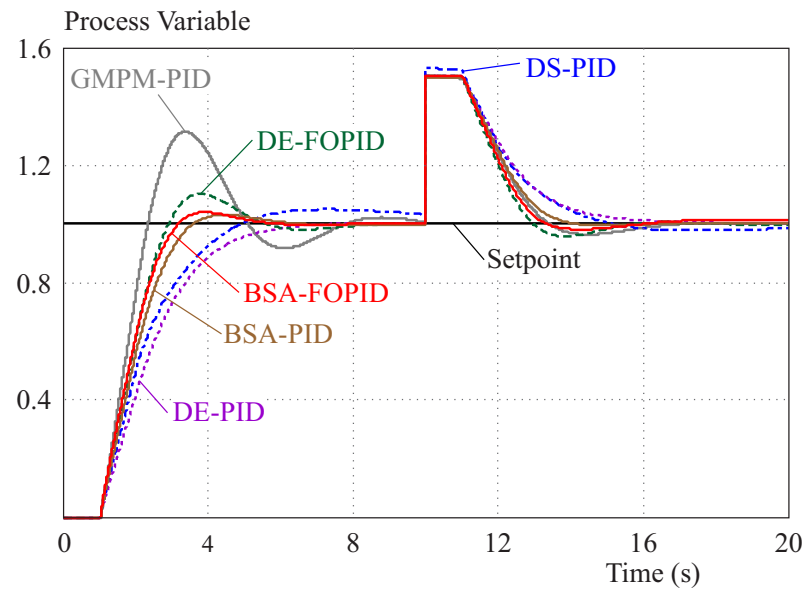

Fig. 7. Process variable variation for $5 \%$ change in dead time

Two different cases have been considered in which it is changed by $5 \%$ and $10 \%$ respectively. The considered transfer functions for a $5 \%$ or $10 \%$ change in dead time are

$$
G_{m}(s)=\frac{2}{3 s+1} e^{-s} \neq G_{p}(s)=\frac{2}{3 s+1} e^{-\alpha s}
$$

with $\alpha=1.05$ or $\alpha=1.1$.

This investigation evaluates setpoint tracking and regulation abilities. For first case, the variation in process variable is shown in Fig. 7. It may be observed that again the existing solution ie GM-PM tuned PCS controller is providing highest OS and is not able to cope up with this uncertainty in dead time. However, FOPCS is providing adequate response in both servo and regulatory regions. For servo response, it may be noted that the ITAEST value is only 2.213 which is minimum among all the considered controllers. Further, the BSA tuned FOPCS objective function is also minimum and stands at only 3.813 in comparison of existing PCS structure tuned using GM-PM method [1], which is providing a objective function value of 6.371 . This comparison clearly indicates that the response of the FOPCS is providing a high degree of robustness in this investigation also. Table 6 presents a quantitative comparison among all the considered controllers.

A similar study is also conducted for $10 \%$ change in process dead time and for the sake of brevity of the paper the graphs have been omitted from this paper. The quantitative assessment of performance of the considered controller is depicted in Tab. 7 and it reveals that the BSA tuned FOPCS controller is providing least objective function value along with optimal IARCO value.

It may be noted here that the objective function value for BSA tuned PCS and BSA tuned FOPCS is almost same. However, the performance of the formed control structure is achieved on the basis of the increased aggression in controller action. The value of the IARCO for BSA tuned PCS controller is at 158.626 in comparison of only 4.299 for BSA tuned FOPCS controller. This is a significant improvement of FOPCS over conventional
PCS, that for similar level of performance the control action does not need to change aggressively which reduces the risk of wear and tear of the control elements in the loop.

Table 7. Performance comparison of controllers for $10 \%$ change in dead time

\begin{tabular}{lccccc}
\hline Method & ITAE $_{\text {ST }}$ & ITAE $_{\mathrm{DR}}$ & OS $(\%)$ & IARCO & $J$ \\
\hline GMPM-PID & 4.939 & 1.723 & 33.796 & 2.891 & 7.000 \\
DS-PID & 4.684 & 2.434 & 5.246 & 685.419 & 7.171 \\
DE-PID & 3.857 & 1.886 & 0.207 & 201.068 & 5.746 \\
DE-FOPID & 3.187 & 1.511 & 12.569 & 2.765 & 4.82 \\
BSA-PID & 2.852 & 1.435 & 4.721 & 158.626 & 4.34 \\
BSA-FOPID & 2.535 & 1.743 & 6.115 & 4.299 & 4.340 \\
\hline
\end{tabular}

Also, it may be observed from all the conducted studies that the FOPCS control structure has performed well for all the considered investigations. The conventional PCS using an integer order PID controller is not capable of providing a better performance in case of robustness, as when there is plant model mismatch, the controller's performance deteriorates. This was also verified by putting different gains achieved through different tuning methods. Neither conventional nor global optimization techniques tuned conventional control structures viz. GMPM-PID [1], DS-PID, DE-PID, or BSA PID was able to provide a satisfactory level of performance. However, with the inclusion of fractional order calculus in the system, the level of robustness was significantly increased. Also for all the considered controllers, it was observed that the FOPCS tuned by BSA provided best performance on the basis of objective function value. Further, it may also be noted that FOPCS tuned by BSA was able to provide a low level of IARCO value.

\section{Conclusion}

The robustness requirement in parallel control structure (PCS) can be achieved through different tuning methods, but at the expense of reduced control performance. To address this issue, this paper investigates a modified parallel control structure which incorporates the benefits of fractional order calculus for enhancing the robustness without compromising with the control performance. For evaluating and comparing the performance of fractional order parallel control structure (FOPCS) with conventional PCS, extensive simulation studies for a first order plus dead time system were considered. These simulation studies include setpoint tracking and disturbance rejection for nominal as well as plant - model mismatch case. Both PCS and FOPCS were tuned using different optimization techniques viz . gain margin - phase margin (GM-PM) method, direct synthesis method, differential evolution method, and backtracking search algorithm. The performance criteria was taken as a composite objective function index $i e$ algebraic summation of integral 
of absolute error for setpoint tracking ( ITAE $_{\mathrm{ST}}$ ), integral of absolute error for disturbance rejection (ITAEDR) and OS value. Based on the obtained results, it may be concluded that BSA tuned FOPCS was able to provide minimum objective function value among all the considered controllers and provided at least $26.28 \%$ improvement over existing PCS tuned by GM-PM method. Another improvement of FOPCS is the reduced level of integral of absolute rate of controller output (IARCO) even for a better level of control performance. Overall, it may be concluded that a properly tuned PCS assisted by fractional order calculus will result in improved level of robustness without any compromise with the control performance.

\section{REFERENCES}

[1] G. Karunagaran and C. Wenjian, "The Parallel Control Structure for Transparent Online Tuning", Journal of Process Control, vol. 21, no. 7, pp. 1072-1079, 2011.

[2] K. J. Åström and T. Hägglund, "The Future of PID Control", Control Engineering Practice,vol. 9, no. 11, pp. 1163-1175, 2001.

[3] L. Desborough and R. Miller, "Increasing Customer Value of Industrial Control Performance Monitoring-Honeywell's Experience", In AIChE Symposium Series 2002, pp. 169-189, New York, American Institute of Chemical Engineers.

[4] P. V. Overschee and B. D. Moor, "The End of Heuristic PID Tuning", IFAC Proceedings, vol. 33, no. 4, pp. 595-600, 2000.

[5] M. Miccio and B. Cosenza, "Control of a Distillation Column by Type-2 Type-1 Fuzzy Logic PID Controllers", Journal of Process Control, vol. 24, no. 5, pp. 475-484, 2014.

[6] P. Mishra, V. Kumar, and K. P. S. Rana, "A Fractional Order Fuzzy PID Controller for Binary Distillation Column Control", Expert Systems with Applications, vol. 42, no. 22, pp. 8533-8549, 2015.

[7] Y. Chen, H. Dou, B. M. Vinagre, and C. A. Monje, "A Robust Tuning Method for Fractional Order PI Controllers", IFAC Proceedings, vol. 39 , no. 11, pp. 22-27, 2006.

[8] A. Oustaloup, F. Levron, B. Mathieu, and F. M. Nanot, "Frequency-Band Complex Noninteger Differentiator: Characterization Synthesis", IEEE Transactions on Circuits Systems I: Fundamental Theory Applications, vol. 47, no. 1, pp. 25-39, 2000.

[9] M. Zamani, M. Karimi-Ghartemani, and N. Sadati, "FOPID Controller Design for Robust Performance Using Particle Swarm Optimization", Fractional Calculus Applied Analysis, vol. 10, no. 2, pp. 169-187, 2007.

[10] V. Kumar, K. P. S. Rana, J. Kumar, and P. Mishra, "Self-Tuned Robust Fractional Order Fuzzy PD Controller for Uncertain Nonlinear Active Suspension System", Neural Computing Applications, pp. 1-17, 2016.

[11] I. Pan and S. Das, "Chaotic Multi-Objective Optimization Based Design of Fractional Order $\mathrm{PI}^{\lambda} \mathrm{D}^{\mu}$ Controller in AVR System", International Journal of Electrical Power \& Energy Systems, vol. 43, no. 1, pp. 393-407, 2012.

[12] I. Pan and S. Das, "Fractional Order AGC for Distributed Energy Resources Using Robust Optimization", IEEE Transactions on Smart Grid, vol. 7, no. 5, pp. 2175-2186, 2016.

[13] I. Pan, S. Das, and A. Gupta, "Tuning of an Optimal Fuzzy PID Controller with Stochastic Algorithms for Networked Control Systems with Random Time Delay", ISA Transactions, vol. 50, no. 1, pp. 28-36, 2011.

[14] S. Das, I. Pan, and S. Das, "Performance Comparison of Optimal Fractional Order Hybrid Fuzzy PID Controllers for Handling
Oscillatory Fractional Order Processes with Dead Time", ISA Transactions, vol. 52, no. 4, pp. 550-566, 2013.

15] V. Kumar, K. P. S. Rana, and P. Mishra, "Robust Speed Control of Hybrid Electric Vehicle using Fractional Order Fuzzy PD PI Controllers in Cascade Control Loop", Journal of the Franklin Institute, vol. 353, no. 8, pp. 1713-1741, 2016.

16] I. Podlubny, "Fractional-Order Systems Fractional-Order Controllers", Institute of Experimental Physics, Slovak Academy of Sciences, Košice,, vol. 12, no. 3, pp. 1-18, 1994.

17] P. Shah and S. Agashe, "Design Optimization of Fractional PID Controller for Higher Order Control System", International conference of IEEE ICART, pp. 588-592 (2013).

18] V. Feliu-Batlle, R. Rivas-Perez, and F. J. Castillo-Garcia, "Fractional Order Controller Robust to Time Delay Variations for Water Distribution in an Irrigation Main Canal Pool", Computers electronics in agriculture, vol. 69, no. 2, pp. 185-197, 2009.

[19] I. Pan, S. Das, and A. Gupta, "Handling Packet Dropouts Random Delays for Unstable Delayed Processes in NCS by Optimal Tuning of Controllers with Evolutionary Algorithms", ISA transactions, vol. 50, no. 4, pp. 557-572, 2011.

[20] Y. C. Cheng and C. Hwang, "Stabilization of Unstable First Order Time Delay Systems using Fractional Order PD Controllers", Journal of the Chinese Institute of Engineers, vol. 29, no. 2, pp. 241-249, 2006.

21] S. Manabe, "Early Development of Fractional Order Control", InASME 2003 International Design Engineering Technical Conferences Computers Information in Engineering Conference 2003, pp. 609-616.

[22] S. Manabe, "A Suggestion of Fractional-Order Controller for Flexible Spacecraft Attitude Control", Nonlinear Dynamics, vol. 29, no. 1, pp. 251-268, 2002.

[23] V. Goyal, P. Mishra, and V. K. Deolia, "A Robust Fractional Order Parallel Control Structure for Flow Control using a Pneumatic Control Valve with Nonlinear Uncertain Dynamics", Arabian Journal for Science Engineering, pp. 1-15, 2018, DOI:10.1007/s13369-018-3328-6.

[24] R. Storn and K. Price, "Differential Evolution - Simple Efficient Heuristic for Global Optimization over Continuous Spaces", Journal of global optimization, vol. 11, no. 4, pp. 341-359, 1997.

[25] S. Panda, "Differential Evolution Algorithm for SSSC-based Damping Controller Design Considering Time Delay", Journal of the Franklin Institute, vol. 348, no. 8, pp. 1903-1926, 2011.

[26] S. Panda, "Robust Coordinated Design of Multiple Multi-Type Damping Controller using Differential Evolution Algorithm", International Journal of Electrical Power \& Energy Systems, vol. 33, no. 4, pp. 1018-1030, 2011.

[27] P. Civicioglu, "Backtracking Search Optimization Algorithm for Numerical Optimization Problems", Applied Mathematics Computation, vol. 219, no. 15, pp. 8121-8144, 2013.

28] D. Guha, P. K. Roy, and S. Banerjee, "Application of Backtracking Search Algorithm in Load Frequency Control of Multi-Area Interconnected Power System", Ain Shams Engineering Journal 2016, (in press, available online).

[29] U. K. Rout, R. K. Sahu, and S. Panda, "Design Analysis of Differential Evolution Algorithm Based Automatic Generation Control for Interconnected Power System", Ain Shams Engineering Journal, vol. 4, no. 3, pp. 409-421, 2013.

[30] Y. Sheoran, V. Kumar, K. P. S. Rana, P. Mishra, J. Kumar, and S. S. Nair, "Development of Backtracking Search Optimization Algorithm Toolkit in LabVIEW ${ }^{\mathrm{TM}}$ ", Procedia Computer Science, vol. 57, pp. 241-248, 2015.

Received 23 November 2018 